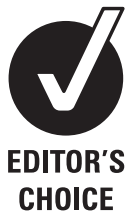

CHOICE

\title{
Endoscopy and implantable electronic devices
}

\author{
G D Corbett, ${ }^{1}$ P C Buttery, ${ }^{2}$ P J Pugh, ${ }^{3}$ E A B Cameron ${ }^{1}$
}

\author{
'Department of \\ Gastroenterology, Addenbrooke's \\ Hospital, Cambridge University \\ Hospitals NHS Foundation Trust, \\ Cambridge, UK \\ 2Department of Neurology, \\ Addenbrooke's Hospital, \\ Cambridge University Hospitals \\ NHS Foundation Trust, \\ Cambridge, UK \\ ${ }^{3}$ Department of Cardiology, \\ Addenbrooke's Hospital, \\ Cambridge University Hospitals \\ NHS Foundation Trust, \\ Cambridge, UK
}

\section{Correspondence to}

Dr Ewen Cameron, Department of Gastroenterology,

Addenbrooke's Hospital, Cambridge University Hospitals NHS Foundation Trust, Box 293 Endoscopy Unit, Cambridge CB2 OQQ, UK;

ewen.cameron@addenbrookes. nhs.uk

Received 25 April 2011 Accepted 10 January 2012 Published Online First

5 February 2012

\begin{abstract}
The increasing use of implantable electronic devices such as cardiac pacemakers and neurostimulators means that they are being increasingly encountered in endoscopy departments. The electromagnetic fields generated during electrosurgery and with magnetic imaging systems have the potential to interfere with such devices. The authors present a case that highlights some of the steps necessary for minimising risk, review the evidence and summarise the currently available guidance.
\end{abstract}

\section{Case}

A 70-year-old female patient attended for a colonoscopy as part of the Bowel Cancer Screening Programme. She suffered from benign essential tremor and had undergone implantation of a ventral intermediate nucleus stimulator device for controlling her tremor. At colonoscopy, she was found to have a $1 \mathrm{~cm}$ pedunculated polyp in the sigmoid colon and a 3 $\mathrm{cm}$ sessile adenoma at the hepatic flexure (figure 1) both of which were biopsied and found to be tubular adenomas with mild to moderate dysplasia. The patient returned for a repeat procedure with piecemeal endoscopic mucosal resection of the hepatic flexure lesion and snare polypectomy of the sigmoid lesion. A consultant neurologist attended to allow the ventral intermediate nucleus stimulator to be disabled during diathermy and checked postprocedure. The procedure was uncomplicated and there has been no evidence of recurrent adenoma at 3 or 12 months (figure 2).

\section{Discussion}

Patients with implantable electronic devices are encountered with increasing frequency by endoscopists as a result of their widespread use. For example, annual permanent pacemaker placements in the UK increased over a decade from 28851 in 1998 to 36297 in 2007 and implantable cardioverter defibrillators (ICDs) from 936 to $3762 .{ }^{1}$ Other devices which may be encountered include neurological stimulator devices and implanted infusion pumps.

\section{Why is there a risk?}

Implantable electronic devices may be susceptible to electromagnetic interference, the effect of an electromagnetic field (EMF) from another source on the device. ${ }^{2}$ During endoscopy, this can either occur when the source comes into direct contact with the patient (conducted; eg, diathermy) or when the body is placed within the field (radiated; eg, with endoscope imaging devices).

The current produced by diathermy inevitably results in an EMF which may lead to implantable electronic device interference. Endoscopic electrosurgical techniques include monopolar and bipolar diathermy currents and argon plasma coagulation.

An EMF can theoretically cause harm by directly affecting the device itself through inhibition or triggering, returning the device to manufacturer presets, causing direct damage to the device or loss of battery power or via the leads at the target site through local heating or uncontrolled stimulation. ${ }^{3}$ The risk of these adverse effects is related to power setting, waveform and frequency (higher risk with cutting than coagulation current), distance from device (return electrode should be placed as far as possible from the implantable device) and type of electrocautery (higher risk with monopolar electrocautery due to production of a larger EMF). ${ }^{45}$

There is a paucity of evidence of harm for those undergoing endoscopic procedures, the majority coming from diathermy at open surgery. Although open 


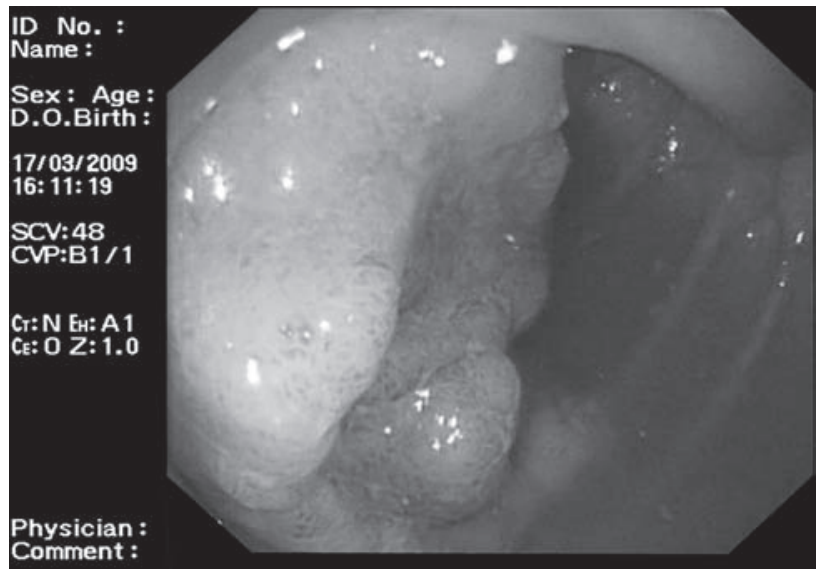

Figure 1 Image of hepatic flexure sessile adenoma.

surgical procedures may be longer and the power setting slightly higher, any electromagnetic interference could cause malfunction in an implantable device and this is relevant to endoscopic electrosurgery.

The manufacturer of ScopeGuide advises against its use in patients with implantable pacemakers. This device produces a low-level magnetic field that could potentially affect the magnetic switch of a pacemaker, possibly resulting in asynchronous pacing or placing the device into a temporary diagnostics mode.

Video capsule endoscopy also produces an EMF. This is the result of the capsule transmitting the images it takes to the recording device. The manufacturers of video capsule systems specifically contraindicate their use with implantable devices. However, a study of 100 patients with pacemakers and a simulator that reproduced the EMF from a video capsule demonstrated no clinically significant effects, although four of the patients' pacemakers did detect interference and resulted in the devices entering asynchronous mode. ${ }^{6}$

\section{Specific Devices and Safety Issues}

\section{Neurostimulators}

Implantable neurostimulators deliver electrical stimulation to target nerve tissue to obtain a therapeutic effect. These devices generally consist of an electrical source, extension wires and one or more leads providing stimulation. Most are controllable remotely via external control modules allowing settings to be altered. Target areas for therapy include brain, spinal cord, neural plexi, stomach and bladder.

Deep brain stimulators were developed to treat Parkinson's disease and other movement disorders by stimulation of the appropriate subthalamic nuclei. ${ }^{7}$ Placement of the device involves stereotactic positioning of the brain electrodes, followed by subcutaneous tunnelling of the lead posterior to the ear. Extension wires are then tunnelled to the anterior chest wall where the pulse generator itself is most frequently inserted.

There are few safety data regarding the impact of electrosurgery on neurostimulator devices. In addition to resetting of parameters, potential risks include

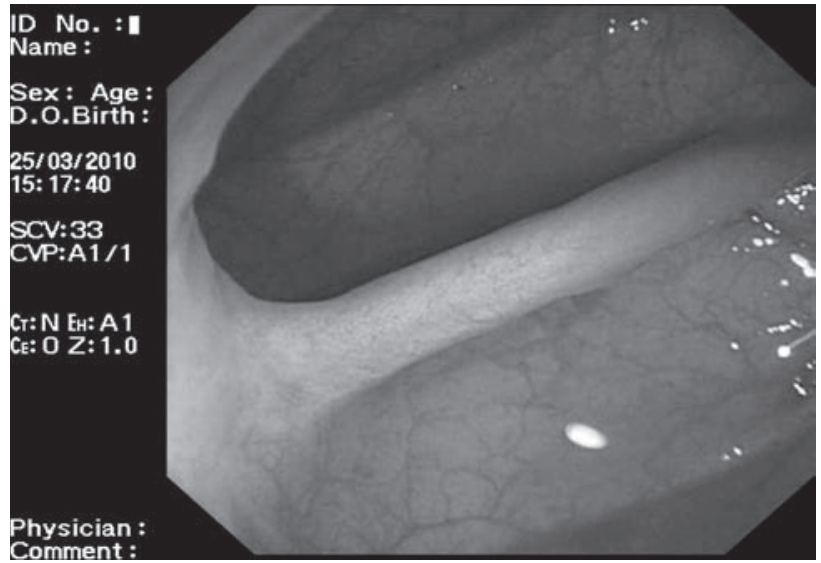

Figure 2 Image of hepatic flexure endoscopic mucosal resection site at 12 months.

induction of currents and lead heating which may not be prevented by inactivating the device. A small number of case reports of adverse events secondary to diathermy and MRI have been reported in patients with neurostimulators; examples include reversible focal neurological deficit, seizures and permanent severe central nervous system damage due to lead heating. ${ }^{8-12}$ For MRI, the biggest risk is of component heating with resultant tissue destruction; in the worse case, this can result in coma, paralysis or death. Manufacturers typically recommend avoiding monopolar diathermy and give strong cautions against MRI except for specific coils and settings. ${ }^{13}$

\section{Implantable Cardiac Devices}

Implantable cardiac devices include pacemakers or ICDs. These are generally implanted into the anterior left prepectoral region. The device is then linked to the heart with pacing leads that pace and sense.

Cardiac pacemakers may be programmed using multiple variables but generally fall into either 'asynchronous' or 'synchronous' modes. The former provides pacing without sensing cardiac activity, whereas the latter senses atrial and/or ventricular activity to determine pacing requirement. Some patients reliant on pacing to maintain cardiac output are referred to as being 'pacemaker dependent'. ${ }^{14}$

ICDs are used in patients at high risk of developing life-threatening ventricular arrhythmias. These devices can also provide bradycardia pacing and cardiac resynchronisation therapy. Sensing of ventricular rate is the key and this function is usually performed via an electrode, implanted within the right ventricle. The device can deliver therapy according to three strategies: antitachycardia pacing for ventricular tachycardia (VT), countershocks for ventricular fibrillation (VF), or VT and antibradycardia pacing if necessary following cardioversion or for symptomatic bradycardia/asystole.

The sensing threshold of pacemakers is usually programmed at $2 \mathrm{mV}$, well above those of the EMFs produced during diathermy. ${ }^{15}$ In contrast, the sensing threshold of ICDs is set lower, at around $0.3 \mathrm{mV}$ in 
Table 1 Summary of Medicines and Health Regulatory Authority guidance for perioperative care of patients with implantable electronic devices where the use of surgical diathermy is anticipated

\begin{tabular}{ll}
\hline Preoperative & Intraoperative \\
\hline $\begin{array}{l}\text { Identify patients through preadmission screening } \\
\begin{array}{l}\text { Contact the patient's pacemaker/ICD clinic to confirm the cardiac } \\
\text { condition and confirm device details }\end{array}\end{array}$ & $\begin{array}{l}\text { CPR facilities and specialist cardiac staff should be available } \\
\text { The patient should be monitored using ECG plus a second method of confirming } \\
\text { heart rate (such as pulse oximetry or arterial line) } \\
\text { Iiaise with device follow-up clinic to advise on required support by } \\
\text { cardiac pacing/ICD physiologist before, during or after surgery }\end{array}$ \\
$\begin{array}{ll}\text { An appointment is made to ensure device functionality after surgery } \\
\text { Minimise monopolar diathermy to short bursts }\end{array}$ \\
$\begin{array}{l}\text { Ensure the return electrode is positioned so that the current pathway is as far from } \\
\text { the device as possible } \\
\text { ICDs should be deactivated and the patient fitted with external defibrillator pads if } \\
\text { access to the anterior chest would interfere with surgery }\end{array}$ \\
\hline
\end{tabular}

ICD, implantable cardioverter defibrillators; CPR, Cardiopulmonary resuscitation.

Table 2 Preinvestigation and postinvestigation recommendations for patients with implantable devices undergoing VCE

\begin{tabular}{lll}
\hline & Preprocedure & Postprocedure \\
\hline Pacemakers & Check pacemaker. & Check pacemaker. \\
& Pacing dependent patients: programme to & Reprogramme to synchronous mode if \\
& asynchronous mode. & necessary. \\
ICDs & Disable device delivered therapies. & Check device function. \\
& Keep patient under observation during passage of VCE. & Reprogramme to enable therapies. \\
\hline
\end{tabular}

ICD, implantable cardioverter defibrillator; VCE, video capsule endoscopy.

order to detect the low-amplitude signals of VF. The data regarding the specific safety of endoscopic diathermy in patients with cardiac pacemakers are anecdotal. Two series have reported no evidence of harm without precautions. ${ }^{16} 17$

Pacing inhibition and triggering, mode switching, incorrect detection of tachyarrhythmia, device reset, myocardial burns, VF and death have been reported in patients undergoing surgical procedures with diathermy. ${ }^{18-20}$ In patients who are 'pacemaker dependent' with sensing pacemakers, endoscopists should be aware of the theoretical risk that delivery of diathermy could be interpreted as cardiac activity and result in pacing inhibition, leading to bradycardia or even asystole.

ICDs could theoretically discharge during diathermy as the waveform may be 'sensed' as a ventricular arrhythmia. However, there is little evidence of this in the literature. One series of 48 patients with ICDs in whom electrocautery was used found no device related complications. ${ }^{21}$ Another paper published a survey of 166 cutaneous surgeons who reported four episodes of ICD discharge in association with electrosurgery. ${ }^{22}$

\section{Infusion Pumps}

Implantable infusion pumps are used for patients requiring continuous parenteral drug administration (eg, baclofen, insulin, opioids and local anaesthetics). These provide a constant rate of infusion (which may be programmable) and are also able to store the agent (usually refilled using a skin port). The infusion may be intravascular, intrathecal or epidural.

There are no case reports of complications associated with electrosurgery and infusion pumps in the literature; however, there is a theoretical risk of battery depletion and device discharge, and one manufacturer has issued specific safety guidance. ${ }^{23}$ However, the United States Food and Drug Administration have over 150 reports of device malfunction in association with electromagnetic interference. These are almost all related to MRI scanning and the commonest fault was pump failure. ${ }^{24}$

\section{Published Safety Guidelines}

In the UK, there are currently no specific guidelines pertaining to endoscopy in patients with implantable electronic devices; however, the Medicines and Health Regulatory Authority published guidance for perioperative care of patients with implantable cardiac devices $^{25}$ (summarised in table 1 ).

In emergency situations, it may be necessary to proceed without standard checks. Securing a clinical magnet over an ICD may prevent inappropriate shock delivery although the response of these devices and pacemakers will depend on how the device has been programmed. If this method is used, external defibrillation will be required for VT or VF and the device must be checked postprocedurally.

The American Society of Gastrointestinal Endoscopists has also provided detailed guidance 
for managing patients with implantable devices who are having endoscopic procedures. ${ }^{26}$ The guidance acknowledges that there are limited data demonstrating complications during endoscopic procedures; however, the potential life-threatening nature of the risk justifies the use of various strategies to minimise this risk.

There are no published guidelines regarding patients with implantable devices undergoing video capsule endoscopy. The absolute contraindication recommended by the manufacturers is not backed by evidence as described above. Until nationally accepted guidelines are published, pragmatic recommendations are described in table 2.

\section{Conclusions}

Endoscopists should be aware of the potential risks associated with the use of diathermy, endoscope magnetic imaging devices and video capsule endoscopes in patients with implantable electronic devices, particularly in view of the increasing array of devices and frequency with which they are encountered. These risks can be minimised by preprocedural preparation, liaison with the appropriate specialists, optimal selection of diathermy current and return electrode positioning.

Contributors GDC and EABC conceived, designed and drafted the article. PCB and PJB revised the article. All authors approved the final version.

\section{Competing interests None.}

Patient consent Obtained.

Provenance and peer review Not commissioned; externally peer reviewed.

\section{References}

1. CCAD, Commissioned by the Healthcare Quality Improvement Partnership. Report for European Heart Rhythm Association, UK. 2007. http://www.ic.nhs.uk/ webfiles/Services/NCASP/audits\%20and\%20reports/ Annual\%20Report\%202007.pdf (accessed 29 Jan 2012).

2. Sager DP. Current facts on pacemaker electromagnetic interference and their application to clinical care. Heart Lung 1987;16:211-21.

3. Madigan JD, Choudhri AF, Chen J, et al. Surgical management of the patient with an implanted cardiac device: implications of electromagnetic interference. Ann Surg 1999;230:639-47.

4. Pinski SL, Trohman RG. Interference in implanted cardiac devices, Part I. Pacing Clin Electrophysiol 2002;25:1367-81.

5. Pinski SL, Trohman RG. Interference in implanted cardiac devices, part II. Pacing Clin Electrophysiol 2002;25:1496-509.

6. Dubner S, Dubner Y, Gallino S, et al. Electromagnetic interference with implantable cardiac pacemakers by video capsule. Gastrointest Endosc 2005;61:250-4.

7. Koller W, Pahwa R, Busenbark K, et al. High-frequency unilateral thalamic stimulation in the treatment of essential and parkinsonian tremor. Ann Neurol 1997;42:292-9.

8. Weaver J, Kim SJ, Lee MH, et al. Cutaneous electrosurgery in a patient with a deep brain stimulator. Dermatol Surg 1999;25:415-17.
9. US Food and Drug Administration. Adverse event report. Available at: http://www.accessdata.fda.gov/scripts/cdrh/ cfdocs/cfmaude/detail.cfm?mdrfoi_id $=349768 /$ (accessed 29 Jan 2012).

10. Nutt JG, Anderson VC, Peacock JH, et al. DBS and diathermy interaction induces severe CNS damage. Neurology 2001;56:1384-6.

11. Roark C, Whicher S, Abosch A. Reversible neurological symptoms caused by diathermy in a patient with deep brain stimulators: case report. Neurosurgery 2008;62:E256; discussion E256.

12. Henderson JM, Tkach J, Phillips M, et al. Permanent neurological deficit related to magnetic resonance imaging in a patient with implanted deep brain stimulation electrodes for Parkinson's disease: case report. Neurosurgery 2005;57:E1063; discussion E1063.

13. Medtronic DBS MRI guidelines. http://professional.medtronic. com/wcm/groups/mdtcom_sg/@mdt/@neuro/documents/ documents/dbs-2007-mri.pdf/ (accessed 29 Jan 2012).

14. Vardas PE, Auricchio A, Blanc JJ, et al. Guidelines for cardiac pacing and cardiac resynchronization therapy: the task force for cardiac pacing and cardiac resynchronization therapy of the european society of cardiology. Developed in collaboration with the European Heart Rhythm Association. Eur Heart J 2007;28:2256-95.

15. Levine PA, Balady GJ, Lazar HL, et al. Electrocautery and pacemakers: management of the paced patient subject to electrocautery. Ann Thorac Surg 1986;41:313-17.

16. Ito S, Shibata H, Okahisa T, et al. Endoscopic therapy using monopolar and bipolar snare with a high-frequency current in patients with a pacemaker. Endoscopy 1994;26:270.

17. Tanigawa K, Yamashita S, Maeda Y, et al. Endoscopic polypectomy for pacemaker patients. Chin Med J 1995;108:579-81.

18. Casavant D, Haffajee C, Stevens S, et al. Aborted implantable cardioverter defibrillator shock during facial electrosurgery. Pacing Clin Electrophysiol 1998;21:1325-6.

19. Geddes LA, Tacker WA, Cabler P. A new electrical hazard associated with the electrocautery. Med Instrum 1975;9:112-13.

20. Nercessian OA, Wu H, Nazarian D, et al. Intraoperative pacemaker dysfunction caused by the use of electrocautery during a total hip arthroplasty. J Arthroplasty 1998;13:599-602.

21. Fiek M, Dorwarth U, Durchlaub I, et al. Application of radiofrequency energy in surgical and interventional procedures: are there interactions with ICDs? Pacing Clin Electrophysiol 2004;27:293-8.

22. El-Gamal HM, Dufresne RG, Saddler K. Electrosurgery, pacemakers and ICDs: a survey of precautions and complications experienced by cutaneous surgeons. Dermatol Surg 2001;27:385-90.

23. Synchromed II pump. System Components Clinical Reference Manual. Minneapolis: Medtronic 2004:11.

24. United States FDA, Manufacturer and User Facility Device Experience. http://www.accessdata.fda.gov/scripts/cdrh/cfdocs/ cfmaude/search.cfm (accessed 29 Jan 2012).

25. Medicines and Healthcare products Regulatory Authority. Guidelines for the perioperative management of patients with implantable pacemakers or implantable cardioverter defibrillators, where the use of surgical diathermy/ electrocautery is anticipated, 2006.

26. Petersen BT, Hussain N, Marine JE, et al. Endoscopy in patients with implanted electronic devices. Gastrointest Endosc 2007;65:561-8. 